\title{
Controlled Laser Texturing of Titanium Results in Reliable Osteointegration
}

Melanie J Coathup BSc, $\mathrm{PhD}^{1}$, Gordon W Blunn BSc, $\mathrm{PhD}^{1}$, Nazanin Mirhosseini BSc, $\mathrm{PhD}^{2}$, Karen Erskine BSc, MBBS ${ }^{1}$, Zhu Liu BSc, $\mathrm{PhD}^{3}$, David R Garrod MA, PhD, FRSA ${ }^{4}$, Lin Li BSc, PhD, FREng ${ }^{2}$

${ }^{1}$ Institute of Orthopaedics and Musculoskeletal Science, University College London, The Royal National Orthopaedic Hospital, UK.

2. Laser Processing Research Centre, School of Mechanical, Aerospace and Civil Engineering, University of Manchester, UK

${ }^{3 .}$ School of Materials, University of Manchester, UK

${ }^{4}$ Faculty of Life Sciences, University of Manchester, UK

\section{Corresponding author and author for reprint requests.}

Dr. Melanie Coathup

${ }^{1}$ Institute of Orthopaedics and Musculoskeletal Science, University College London, The Royal National Orthopaedic Hospital, United Kingdom. Tel: +44 208954 5264, Fax: +44 208420 7392, email: m.coathup@ucl.ac.uk

Running Title: Osteointegration of Laser Textured Implants 


\title{
Author Contributions:
}

The authors Li, Blunn, Garrod and Liu contributed to the study design. Authors Li, Garrod, Mirhosseini and Liu prepared the laser-etched surfaces. Animal surgery, data acquisition, analysis and interpretation of data was performed by Blunn, Erskine and Coathup. The manuscript was drafted by Coathup and Blunn and all authors have read and approved the final submitted manuscript.

\begin{abstract}
We have developed a laser-textured superhydrophilic Ti-6Al-4V surface with unique surface chemistry and topography that substantially promotes osteoblast adhesion in culture. Here we investigate the osteointegration of laser-textured implants in an ovine model. Our hypothesis was that laser-textured implants, without any surface coating (LT), would encourage comparable amounts of bone-implant contact and interfacial strength when compared with widely accepted hydroxyapatite (HA) coated implants. Additionally, we hypothesized that LT would significantly increase bony integration compared with machine-finished (MF) and grit-blasted (GB) implants. Forty-eight tapered transcortical pins were implanted into six sheep. Four experimental groups (LT, HA, MF and GB) were investigated(n = 12) and implants remained in vivo for 6 weeks. Bone apposition rates, interfacial shear strength and bone-implant contact (BIC) were quantified. The interfacial strength of LT and HA implants were found to be significantly greater than GB ( $p=0.032$ and $p=0.004)$ and MF ( $p=0.004$ and $p=0.004$ respectively), but no significant difference between LT and HA implants was observed. Significantly increased BIC was measured adjacent to HA implants when compared with both LT and GB implant surfaces $(p=0.022$ and $p=0.006$ respectively). No significant difference was found when LT and GB implants were
\end{abstract}


compared. However, all surface finishes encouraged significantly increased BIC when compared with the MF surface.

Clinical Significance: Maximising implant fixation to host bone is vital for its long-term success. The production of an LT surface is a simple and cheap manufacturing process and this study demonstrated that laser-textured implants are a very promising technical development that warrants further research.

Keywords: Laser-textured; osteointegration, hydroxyapatite; animal model; bone regeneration.

\section{Introduction}

Over 160,000 hip and knee replacements were undertaken in England and Wales in 2011, accounting for a significant proportion of NHS resources (1). Data from an American national database showed that $46 \%$ of total hip arthroplasties (THAs) are now being performed in patients under 65 years of age, and this is predicted to increase to $55 \%$ by 2015 (2). With this change in patient demographics there is a requirement for enhanced implant longevity, which requires optimisation of bone fixation.

Cementless fixation uses surface coatings or textured ongrowth surfaces to invoke new bone growth at the implant interface leading to biological fixation through osteointegration. This biological process permits regeneration and remodelling over time and also allows for the adaptive capability of bone to respond to any changes in the patients' biomechanical environments (3). Implant osteointegration is key to increasing longevity of an uncemented implant in orthopaedic surgery and the choice of ingrowth surface used is crucial as it affects 
long-term survival (4). It has been postulated that a porous structure, has a role in stimulating bone formation and preventing bone resorption (5). Optimum bone growth is associated with a pore size ranging from $200-400 \mu \mathrm{m}$ (and a minimal pore size of 50 to $150 \mu \mathrm{m}$ ) (6). A successful surface coating in terms of achieving osteointegration has involved the addition or alteration in quantities of inorganic phases such as calcium and phosphate (7). Calcium phosphate coatings replicating the mineral phase of bone are bioactive and able to bond directly with bones, thereby increasing implant interfacial strength (8). A study by Coathup et al. (9) investigated uncemented femoral components retrieved from 58 patients at postmortem and compared bone growth to a hydroxyapatite (HA) porous coating with an uncoated plasma-sprayed porous coating and a grit blasted surface finish. This study reported increased and more evenly distributed bone in direct contact with the HA coated porous surface when compared with the porous only and grit blasted surfaces. Animal studies have further supported these findings where HA coated implants increased direct bone-implant contact (BIC) and increased torque by four times when compared with non-coated grit blasted surfaces $(10,11)$. HA coated surfaces have also been shown to be more resistant to migration of wear debris along the interface (12). Despite showing excellent long-term clinical results, there are concerns regarding HA's resorption over time and concomitant exposure of the underlying titanium surface, which is reported to decrease bone-implant contact in the years immediately post-surgery $(13,14)$. Delamination of HA has also been reported for thick coatings where there is a separation between the alloy surface and the plasma sprayed HA surface and this is believed to be associated with the difference in stiffness between these materials. HA particles have been shown to elicit an inflammatory response and are reported to contribute to third-body wear, osteolysis and eventually aseptic loosening (15). 
Various techniques such as grit blast and acid etching have been used to roughen the implant surfaces in order to improve the clinical performance and to encourage a stable mechanical bone-implant interface. However, sand blasting is reported to leave contaminations on the implant surface, which is not desirable (16). On the other hand, both grit blast and acid etch produce surfaces with poorly-defined topography and various chemical compositions and the optimal surface properties which result in rapid osteoblast attachment, proliferation and differentiation are not yet established.

Laser texturing has been developed over the past decade to roughen the implant surface by producing hemispherical pores and this process has been used successfully to augment dental implant fixation (17). The process evaporates the original surface layer and re-shapes a desirable micro morphological topology with enhanced surface roughness and chemical compositions to promote osteointegration. The resulting surface roughness is similar to that produced by acid-texturing and replicates the texture of the bone matrix, and has been shown to enhance osteogenic cells to form new bone $(6,18)$. Single wavelength pulses are fired at a particular spot on the surface, heating the material to a high enough temperature to cause a phase transition. As the material cools again the titanium alloy re-solidifies leaving behind both micro- and nano-indentations. This process modifies the surface topography and wettability without significantly altering its surface chemistry and laser texturing is advantageous to alternative processes as it is site specific, causes less disruption to the titanium's purity and is both clean and repeatable (19).

Various surface textures have been created on the surface of biomaterials including uniform and non-uniform patterns. An example is using a femtosecond and a picosecond laser to create uniform and repeatable patterns on a desired portion of the surface on Ti-6Al-4V. They found that different surface patterns could enhance or inhibit human osteosarcoma cell 
attachment (20). Mirhosseini et al. reported that laser surface patterning of small holes on $\mathrm{Ti}-$ $6 \mathrm{Al}-4 \mathrm{~V}$ samples and the synthesis of $\mathrm{Ca}_{2} \mathrm{SiO}_{4}$ coating on $\mathrm{Ti}-6 \mathrm{Al}-4 \mathrm{~V}$ samples by laser irradiation under submerged conditions would both encourage the 2T3 osteoblast cell growth $(21,22)$. Mukherjee et al studied the effect of laser created grooves with different widths and depths on MG63 cells. They have used a continuous wave fiber laser and showed that surface features dimensionally closer to the cell dimensions were able to positively affect the viability and spreading of MG63 cells (23). Laser surface irradiation of Ti-6Al-4V samples by using a High Power Diode Laser (HPDL) was reported by Hao et al to increase the wettability and osteoblast response (24). Hallgren et al. produced $10 \mu \mathrm{m}$ hemispherical pits with a pitch of 30 $\mu \mathrm{m}$ on titanium dental implants by using a Nd:YAG laser and reported significantly more bone-to-implant contact in rabbit femur and tibia in a twelve week study (25). The above investigations only focused on the laser generation of surface structures and their in vitro effects on biocompatibility, fewer studies have investigated these surfaces in vivo. There are a number of different surfaces available for osteointegration. The advantages of using a laser textured surface are that it produces a surface of intermediate roughness, there is minimal contamination, the porosity that is created is part of the implant and therefore delamination does not occur.

This study aimed to investigate the osteointegration of our novel laser-textured implant in an in-vivo ovine trans-cortical pin model. The laser-textured super-oxidized surface investigated had a novel uniform spaced parallel ridge pattern. Our hypothesis was that implants modified using our laser-texturing technique (LT) would encourage comparable amounts of boneimplant contact and interfacial strength when compared with hydroxyapatite (HA) coated implants. We further hypothesized that an increased amount of bony integration would be 
measured adjacent to LT implants when compared with machine-finished (MF) and gritblasted (GB) implants.

\section{Materials and Methods}

In order to minimise the number of animals required in this study, a power analysis based on our previous work where a significance of $\mathrm{p}=0.05$ showed that the minimum number of animals to achieve a significant difference with a power of 0.8 was 6 in order to observe a $20 \%$ difference between control and experimental groups, which is required for translational/clinical impact. A total of 48 tapered pins measuring 5-4 $\mathrm{mm}$ in diameter and $20 \mathrm{~mm}$ in length were manufactured from titanium alloy (Ti-6Al-4V). Twelve implants were assigned to each of the four experimental groups which were; 1) Grit blasted (GB), 2) Machine-finish (MF), 3) Plasma sprayed HA coated and 4) Laser-textured (LT). All implant surfaces (apart from those in the LT group) were prepared by Plasma Biotal, (Tideswell, Derbyshire, UK). Surface roughness was quantified in all groups except the LT group, using three-dimensional non-contact analyses and Optical Microscopy (Contour-GT, Bruker, MA, USA). Four equi-distant measurements were taken along the length of each of the surfaces and results compared. The MF surface investigated had a mean surface roughness $\left(\mathrm{R}_{\mathrm{a}}\right)$ of 0.1 $\mu \mathrm{m}$, the GB surface $6 \mu \mathrm{m}$ and the HA coated surface a roughness of $2.0 \mu \mathrm{m}$. Implant surfaces in the grit blasted and plasma sprayed HA coated group were blasted with aluminium oxide beads of 5 microns in size. Implants in the plasma sprayed HA group, were additionally coated with a highly crystalline $(>85 \%)$, thin $(<70 \mu \mathrm{m})$ hydroxyapatite coating. Implants were gamma sterilized prior to insertion during surgery, however prior to sterilization and HA coating, implants in all groups were ultrasonically cleaned in Decon 90 ${ }^{\mathrm{TM}}$ (Decon Laboratories Limited, Hove, UK) for 20 minutes, followed by washing in distilled water, isopropanol and trichloroethylene, each for $\mathbf{2 0}$ minutes. Implants were inserted into 6 
skeletally mature, commercially crossbred, female sheep weighing $70-90 \mathrm{~kg}$. A $100 \mathrm{~mm}$ incision was made over the medial aspect of the tibia and the bone exposed using blunt dissection. The periosteum was removed and bi-cortical defects created using a drill and tap prior to implantation of the pin. Implants were separated by at least $1.5 \mathrm{~cm}$ of bone and four pins were inserted into both the left and right tibiae (a total of 8 implants per animal) (Figure 1). Implant positions were rotated such that no implant combination was placed in the same location in each sheep more than once. All procedures were carried out following Ethics approval granted by the Royal Veterinary College and in compliance with the United Kingdom Home Office regulations [Animal Scientific Procedures Act (1986)]. Following surgery, animals were allowed immediate and full mobilization as tolerated. Antibiotic and analgesic prophylaxis were administered daily with subcutaneous injections of Baytril (Enrofloxacin 5 mg/kg; Bayer AG Leverkusen) and Finadyne (Flunixin Meglumine 2 mg/45 $\mathrm{kg}$; Schering-Plough) for 3 days post-surgery. Animals were kept indoors and in individual pens for 1 week post operatively before being group housed. The general health of all animals was observed and recorded daily, and transfer of animals from one housing regime to another was dependent upon recovery from the surgery, and was therefore made on an individual basis. Two fluorochrome bone markers Oxytetracyline $(30 \mathrm{mg} / \mathrm{kg})$ and Calcein Green $(30 \mathrm{mg} / \mathrm{kg}$ ) were administered at weeks 3 and 5 post surgery. Oxytetracylcine and Calcein Green localize at sites of mineralization and, when viewed under ultra-violet light, fluoresce orange and green, respectively. The mean measurable distance between cement lines provided an assessment of bone apposition rates ( $\mu \mathrm{m} /$ day) adjacent to pins in each of the groups. Implants remained in vivo 6 weeks and animals were euthanized following administration of sodium pentobarbital (Euthatal, 150mg/kg; JM Loveridge Plc, Hampshire, UK). This time-point was chosen as it is considered a clinically relevant time-point where bone formation has been shown to occur. 
Within 8 hours of retrieval, the interfacial shear strength of 6 implants in each experimental group were assessed using a Zwick ProLine 500 (Zwick/Roell, LTominster, Herefordshire, UK) pull-out testing machine. The remaining 6 implants in each group, were placed in $4 \%$ paraformaldehyde solution before being processed for undecalcified histology. Following dehydration in serial dilutions of alcohol, specimens were defatted and embedded in hard grade acrylic resin (LR White, London Resin Company, Reading, UK). Thin sections ( 70 $\mu \mathrm{m})$ were prepared by making longitudinal cuts through the centre of each pin using a grinding and polishing technique (EXAKT, Norderstedt, Germany). Samples were stained with Toluidine Blue and Paragon, which stained the soft tissue and bone respectively. Bone contact was assessed along the entire bone-implant interface of each of the pins using image analysis software (Axiovision Release 4.6, Carl Zeiss, Jena, Germany). The ridged surface of the LT implant demonstrated bone growth between the ridges and onto the surface of those ridges. This allowed us to measure the amount of bone between the ridges. Eight random regions of interest (ROI) were image captured along the implant interface and assessments made using image analysis software to quantify the proportion of mineralised bone that had integrated within the structure.

\section{Laser Surface Texturing}

A diode pumped $\mathrm{Nd}: \mathrm{YVO}_{4}$ laser with a wavelength of $532 \mathrm{~nm}$, a pulse duration of $8 \mathrm{~ns}$ and a repetition rate of $30 \mathrm{kHz}$ was used to texture the implants in a $\mathrm{N}_{2}$ gas environment. The laser beam with a near Gaussian intensity distribution $\left(\mathrm{M}^{2} \sim 1.5\right)$ was focused onto the surface with a spot diameter of $\sim 55 \mu \mathrm{m}$.

The laser beam was raster scanned at a speed of $20 \mathrm{~mm} / \mathrm{s}$ over the surface of the implants in parallel scanning lines with a hatch distance of $20 \mu \mathrm{m}$, using a computer controlled galvo- 
scanning system equipped with a flat field lens. This produced a ridge height and width of 56 and $20 \mu \mathrm{m}$ respectively with a spacing of $40 \mu \mathrm{m}$. This also has a structure on the ridges, which has a roughness in the micron range (Figure 2; Table 1).

\section{Interfacial Shear Test}

Interfacial shear strength measurements were carried out in accordance with ISO-13779-4. A M3 thread located within the implant was attached to a Zwick ProLine 500 (Zwick/Roell, Leominster, Herefordshire, UK) testing machine, and a pre-load of $1 \mathrm{~N} / \mathrm{mm}^{2}$ and a test speed of $5 \mathrm{~mm} / \mathrm{min}$ applied. Force-deformation curves were recorded and the maximum force (Fmax) was used to represent the maximum strength of the bone-implant interface.

\section{Backscattered Scanning Electron Microscopy}

Thin sections were sputter-coated with a layer of gold palladium and viewed using Backscattered electron microscopy (JOEL JSM-5500LV; Jeol, Welwyn Garden City, UK).

\section{Statistical Analysis}

Analysis of the data was performed using SPSS software (v10.1; SPSS, Chicago, Illinios, USA). A Kolmogorov-Smirnov test showed the data obtained were non parametric and the Mann-Whitney U test was used for statistical comparison between experimental groups. A Pearson's $r$ correlation coefficient was used to assess the relationship between the mean boneimplant contact (BIC) and the mean interfacial shear strength for each group. Individual implants were unable to be compared as data for both BIC and shear strength was obtained from different implants as measurement techniques were destructive. $P$ values $<0.05$ were considered significant. 


\section{RESULTS}

All animals remained healthy throughout the duration of the study.

\section{Bone Apposition Rates}

Results ( \pm standard deviation (SD)) showed no significant difference when each of the experimental groups were compared. The highest bone apposition rate was measured in the GB group (mean, $1.92 \pm 0.20 \mu \mathrm{m} /$ day) and the lowest was observed adjacent to LT implants (mean, $1.67 \pm 0.22 \mu \mathrm{m} /$ day) (Figure 3). A mean of $1.79 \pm 0.25 \mu \mathrm{m} /$ day was measured in the HA group and $1.93 \pm 0.19 \mu \mathrm{m} /$ day in the MF implant group.

\section{Bone-Implant Contact}

Bone-implant contact adjacent to HA coated pins was significantly increased (mean, $64.85 \pm$ $10.18 \%$ ) when compared with each of the other surface finishes (LT mean, $36.81 \pm 9.61 \%, p$ $=0.022$; GB mean, $36.79 \pm 11.15 \%, p=0.006$; and MF mean, $15.07 \pm 5.61 \%, p=0.006)$. Significantly increased bone-implant contact was measured in the LT and GB groups when compared with the MF surface finish ( $p=0.004$ and 0.006 respectively). No significant difference was found when LT and GB implants were compared (Figure 4).

\section{Bony Ongrowth into the LT Surface}

Due to the nature of the surface, bone ongrowth on LT implants showed that a mean of 48.78 $\pm 6.15 \%$ of the surface had grown on and within the ridges. In the LT group, BSEM showed direct bone growth within the ridges (Figure 5). Osteoconduction along the HA surface was observed where more bone appeared on the HA surface where the pin transected within the medullary canal. This was also seen to a lesser degree in the LT group. Backscattered 
scanning electron microscopy showed that in the MF group, bone growth was seen immediately adjacent to the implants. However in most places, bone did not make direct contact with the surface.

\section{Interfacial Shear Strength}

The maximum force (F-max) sustained before the pin was detached from the surrounding bone was recorded. The highest measure of interfacial shear strength was seen in the HA coated group (mean, 2611.16 $\pm 306.54 \mathrm{~N}$ ) (Figure 6). A significantly higher interfacial strength was measured in the HA group when compared with all groups except the LT group (mean, 2265.26 $\pm 751.23 \mathrm{~N}$; GB mean, $1199.89 \pm 444.77 \mathrm{~N}, p=0.004$; MF mean, $183.05 \pm$ $151.05 \mathrm{~N}, p=0.004)$. A significantly higher interfacial strength was measured in the LT samples when compared with both the GB $(p=0.032)$ and MF $(p=0,004)$ groups. Additionally, significantly increased interfacial shear strength was measured adjacent to GB implants when compared with MF implants $(p=0.004)$. It was evident that failure during pullout testing in the MF and GB groups occurred along the bone-implant interface. Whereas for the LT group, failure occurred within the bone with bone tissue identified within the LT ridges. For the HA implants, failure occurred both within the bone and along the HA-metal interface exposing parts of the underlying titanium alloy (Figure 7)

Correlation analysis showed a strong relationship between BIC and interfacial shear strength $(r=0.88)$ (Figure 8a). Results showed that LT implants fell below the trend and when removed from the analysis. A stronger correlation of $r=0.99$ was found (Figure $8 \mathrm{~b}$ ). This suggested the LT grooved surface increased the force required to remove the implant from bone despite the lower BIC value. 


\section{Histology}

Qualitative analysis using optical microscopy showed that all surface finishes investigated, including MF, demonstrated direct bone contact to the implant surface. However, implants coated with HA showed more continuous bone-implant contact. Although direct boneimplant contact was seen in the GB and LT groups, there were many areas where bone was interrupted by a soft tissue layer interfacing the implant surface (Figure 9). Although osteogenesis had occurred in the MF group, the bone was separated from the underlying titanium alloy surface by a thin layer of fibrous tissue.

\section{DISCUSSION}

The bone-implant interface was investigated both qualitatively and quantitatively using biomechanical testing, histology and backscattered SEM. Results showed a significant increase in the levels of BIC with HA, LT and GB compared to MF, and when HA was compared to both LT and GB. However, our study did not find a significant difference when LT and GB surfaces were compared. These findings were also reflected in the biomechanical test results, with the exception that the interfacial strength at the LT interface significantly increased when compared with GB surfaces. Our results therefore do not support the first part of our hypothesis, that a laser-textured technique would encourage comparable amounts of bone-implant contact when compared with hydroxyapatite (HA) coated implants. However, we can accept part of our second hypothesis as increased bony integration was measured adjacent to LT implants when compared with the machine-finished surface.

Results showed that BIC measured in the HA group (65\%) was almost twice that measured in both the LT (37\%) and GB (37\%) groups. A study by Abeli et al. (26) measured a similar amount of BIC and reported that significantly increased osteointegration occurred adjacent to 
a HA coated implant (68\%) when compared with a non-coated vacuum plasma sprayed titanium implant surface (46\%) in an ovine model at 4 weeks post-implantation. Similarly, an intramedullary rabbit model reported significantly increased BIC (64\%) to a HA coated implant 6 weeks post-implantation when compared with a non-coated chemically-textured titanium surface (39\%) (27). Our pull-out test data is also comparable with studies reported in the literature (28) and Aebli et al. (26) showed HA to have a significantly superior interfacial strength when compared to a GB control. Results from our study also showed no evidence of HA delamination, resorption, or an inflammatory response along the interface whilst the implants were in situ confirmed the effectiveness of HA in bony regeneration and osteointegration when compared with non-coated titanium surfaces. We did, however, show that delamination of the HA coating occurred during pull-out tests. This exposed the underlying alloy surface, which was also exposed when MF and GB implants were pulled from the bone. However, failure within the bone occurred for the LT implants.

Previous studies have concluded that rougher surfaces increase BIC along the interface (6, 29). However, our findings do not support this conclusion as our results showed that mean BIC values were similar in the GB and LT groups. A study by Stangl et al. (30) failed to observe any advantage of using a laser-textured surface with a pore size of $50 \mu \mathrm{m}(14 \%)$ over a grit-blasted surface $(23 \%)$ in a rabbit intramedullary model 6 weeks post implantation. A study by Prodanov et al. (31) also showed no significant difference when bone-to-implant contact to a laser-treated surface $(68 \%, \pm 17)$ was compared with a grit blasted/acid etched surface $(49 \%, \pm 21)$ when inserted in a rabbit model at 8 weeks post-implantation. There are many studies that support increased osteointegration when compared to a MF control. Branemark et al. (32) reported that a LT implant significantly augmented BIC (26.5\%) when compared to the MF control (18\%). Paz et al. (19) similarly found BIC at the LT surface to be 
significantly increased (84\%) when compared with a MF control (35.9\%) following a 3 month implantation period. Although studies by Aparicio et al. (33) and Guo et al. (34) reported a $40 \%$ BIC rate to a MF surface, our results showed lower BIC and were similar to other studies where bone contact measured in the range of $10-20 \%(32,35,36)$. A study by Faeda et al. (37), investigated the interfacial shear strength of LT modified surfaces in a rabbit transcortical pin model. Results showed that 4 weeks post-implantation the laser modified implant was associated with a significantly higher removal torque of $3300 \mathrm{~N} / \mathrm{mm}^{2}$ compared to the MF control (2328 N/mm²). Studies by Branemark et al. (32) and Palmquist et al. (38) also reported that a LT surface augmented osteointegration and a significantly higher removal torque was measured when compared with a MF control at 8 weeks and 6 months respectively post-implantation.

The inconsistency seen between pull-out test results and BIC has also been reported in previous studies. In our study, a Pearson's correlation coefficient of 0.88 was measured when comparing the means of each group. This indicates a strong positive relationship between these parameters and is comparable with previous studies $(39,40)$. However in our study, the relationship of BIC and interfacial strength at the LT interface did not follow this trend with stronger interfaces for a given amount of bone-implant contact being seen with the LT implants compared with the HA, MF and GB groups. In the HA, GB and MF groups, there was a very high correlation between BIC and interfacial shear strength. This showed that for every $1 \%$ of BIC there was a concomitant increase of approximately $40 \mathrm{Nmm}^{2}$. However, in the LT implant group, for every $1 \%$ increase in BIC there was an increase of approximately $60 \mathrm{Nmm}^{2}$. The higher than anticipated interfacial strength given the level of measurable BIC achieved could be due to a number of possibilities. The higher interfacial strength at the LT surface suggests the quality of bone anchorage may have been greater than seen with its 
comparator GB. Bone growth onto the LT surface was measured in our study and results showed direct contact with $48.8 \%$ of the ingrowth surface occupied by bone. The larger amount of bone ingrowth on an LT surface may be associated with a more biological response due to bone formation being enhanced between the ridges. The structural nature of the LT surface allowed bone to interdigitate with the implant leading to greater resistance to shear. It is known that rougher surfaces promote osteogenic cells to form new bone around the implant (41). Cooper et al. (6) described the ability of osteoblastic cells to sense the grooved LT surface with the aid of integrins, which then bind to proteins adsorbed to the titanium surface. It has also been reported that laser-engineered titanium surfaces significantly increase the viability, adhesion and proliferation of osteoblastic cells $(18,42)$. An in vitro study investigated LT discs and measured a decrease in the attachment of early inflammatory cells (38). The authors postulated that this decrease was related to the LT surface replicating similar nanometre features resulting in increased extracellular matrix protein attachment.

Maximising the potential for initial stability and good fixation of an implant to the host bone is vital for its long-term success. In this study the degree and strength of osteointegration associated with different surface implants was assessed using an ovine model. Our study investigated osteointegration in healthy cortical bone using tapered pins. The rationale for this was that this is a comparative study of different surface textures and relative differences can be measured using this approach where variability associated with insertion and cancellous bone structure can be limited. Cortical bone models have been used previously to study the interaction of implants with different materials $(43,44)$. Results showed that HA encouraged significantly increased bone-implant contact when compared with LT, GB and MF surface finishes. In terms of their function, LT samples showed no significant difference in their bone attachment from HA implants and were more strongly attached than either GB 
or MF implants. Although BIC to the HA surface was significantly higher, the increase in surface roughness of the LT implants allowed better bone growth which resisted the forces applied during the pull out tests giving an interfacial strength similar to the smoother HA coated surface. The LT surface did not appear to be more bioactive as a result of changes to surface chemistry and the reasons for this remain unclear. The production of an LT surface is a simple and cheap manufacturing process and this study demonstrated that laser-textured implants are a very promising technical development that warrants further research in larger studies.

\section{Acknowledgements}

Authors from the University of Manchester initiated the study, supplied the laser-textured implants and funded the work. Authors from the Institute of Orthopaedics and Musculoskeletal Science were involved in the study design, data collection, analysis and interpretation. No author has had any other relationships, or has engaged in any other activities that could be perceived to influence what is written in this work.

\section{REFERENCES}

1. Porter M, Borroff M, Gregg P, Howard P, Macgregor A, Tucker K. 2012. National Joint Registry for England and Wales [Online]. Available: www.njrcentre.org.uk.

2. Kurtz S, Ong K, Lau E, Mowat F, Halpern M. 2007. Projections of primary and revision hip and knee arthroplasty in the United States from 2005 to 2030. J Bone Joint Surg [Am] 89A:780-5. 
3. Atsumi M, Park S-H, Wang H-L. 2007. Methods used to assess implant stability: current status. Internat J Oral \& Maxillo Impl 22:743-754.

4. Chao EY, Fuchs B, Rowland CM, Ilstrup DM, Pritchard DJ, Sim FH. 2004. Long-term results of segmental prosthesis fixation by extracortical bone-bridging and ingrowth. J Bone Joint Surg [Am] 86-A:948-55.

5. Cosyn J, Sabzevar MM, De Wilde P, De Rouck T. 2007. Two-piece Implants with Turned Versus Microtextured Collars. J Periodontol 78(9):1657-63.

6. Cooper LF. 2000. A role for surface topography in creating and maintaining bone at titanium endosseous implants. J Prosth Dent 84:522-534.

7. Barrere F, Van Blitterswijk CA, De Groot K. 2006. Bone regeneration: molecular and cellular interactions with calcium phosphate ceramics. Int J Nanomedicine 1:317-32.

8. Dohan Ehrenfest D, Coelho P, Kang B-S, Sul Y-T, Albrektsson T. 2010. Classification of osseointegrated implant surfaces: materials, chemistry and topography. Trends in biotechnology 28:198-206.

9. Coathup MJ, Blunn GW, Flynn N, Williams C, Thomas NP. 2001. A comparison of bone remodelling around hydroxyapatite-coated, porous-coated and grit-blasted hip replacements retrieved at post-mortem. J Bone Joint Surg [Br] 83:118-23. 
10. Eckardt A, Aberman HM, Cantwell HD, Heine J. 2003. Biological fixation of hydroxyapatite-coated versus grit-blasted titanium hip stems: a canine study. Arch Orthop Trauma Surg 123:28-35.

11. Magyar G, Toksvig-Larsen S, Moroni A. 1997. Hydroxyapatite Coating Of Threaded Pins Enhances Fixation. J Bone Joint Surg [Br] 79-B:487-489.

12. Coathup MJ, Blackburn J, Goodship AE, Cunningham JL, Smith T, Blunn GW. 2005. Role of hydroxyapatite coating in resisting wear particle migration and osteolysis around acetabular components. Biomaterials 26(19):4161-9.

13. Bloebaum RD, Beeks D, Dorr LD, Savory CG, Dupont JA, Hofmann AA. 1994. Complications with hydroxyapatite particulate separation in total hip arthroplasty. Clin Orthop Relat Res 19-26.

14. Porter A, Taak P, Hobbs L, Coathup M, Blunn G, Spector M. 2004. Bone bonding to hydroxyapatite and titanium surfaces on femoral stems retrieved from human subjects at autopsy. Biomaterials 25:5199-5208.

15. Harada Y, Wang JT, Doppalapudi VA, Willis AA, Jasty M, Harris WH, Nagase M, Goldring SR. 1996. Differential effects of different forms of hydroxyapatite and hydroxyapatite/tricalcium phosphate particulates on human monocyte/macrophages in vitro. J Biomed Mater Res 31:19-26. 
16. Esposito M, Hirsch JM, Lekholm U, Thomsen P. 1998. Biological factors contributing to failures of osseointegrated oral implants. (II).Etiopathogenesis Eur J Oral Sci 106:721-764.

17. Sul Y-T, Kang B-S, Johansson C, Um H-S, Park C-J, Albrektsson T. 2009. The roles of surface chemistry and topography in the strength and rate of osseointegration of titanium implants in bone. J Biomed Mater Res- A 89:942-950.

18. Cei S, LTgitimo A, Barachini S, Consolini R, Sammartino G, Mattii L, Gabriele M, Graziani F. 2011. Effect of laser micromachining of titanium on viability and responsiveness of osteoblast-like cells. Implant Dent 20:285-291.

19. Paz MA, Alavea JI, Goikoetxea L, Chiussi S, Dazafamas I, Usuan J, Sanchez F, Leaan B. 2011. Biological response of laser macrostructured and oxidized titanium alloy: an in vitro and in vivo study. J Appl Biomater Biomech 9:214-222.

20. Erdogan M, Oktem BH, Kalaycioglu H, Yavas S, Mukhopadhyay PK, Eken K, Ozgoren K, Aykac Y, Tazebay UH, Ilday FO. 2011. Texturing of titanium (Ti6Al4V) medical implant surfaces with M-H-z repetition rate femtosecond and picosecondYb-doped fibre lasers. Optical society of America 19:10986-10996.

21. Mirhosseini N, Crouse PL, Schmidth MJJ, Li L, Garrod D. 2007. Laser surface microtexturing of Ti-6Al-4V substrates for improved cell integration. Applied Surface Science 253(19):7738-7743. 
22. Mirhosseini N, Crouse PL, Li L, Garrod D. 2007. Combined laser/sol-gel synthesis of calcium silicate coating on Ti-6Al-4V substrates for improved cell integration. Applied Surface Science 253(19):7998-8002.

23. Mukherjee S, Dhara S, and Saha P. 2015. Enhancing the biocompatibility of Ti6Al4V implants by laser surface microtexturing: an in vitro study. Int J Adv Manuf Technol 76:5-15.

24. Hao L, Lawrence J, and L. Li. 2005. Manipulation of the osteoblast response to a Ti6Al-4V titanium alloy using a high power diode laser. Applied Surface Science 247:602-606.

25. Hallgren C, Reimers H, Chakarov D, Gold J, Weenerberg A. 2003. An in vivo study of bone response to implants topographically modified by laser micromachining. Biomaterials 24(701-710):701-710.

26. Aebli N, Krebs JR, Stich H, Schawalder P, Walton M, Schwenke D, Gruner H, Gasser B, Theis J-C. 2003. In vivo comparison of the osseointegration of vacuum plasma sprayed titanium- and hydroxyapatite-coated implants. J Biomed Mater Res-A 66:356-363.

27. Hermida J, Bergula A, Dimaano F, Hawkins M, Colwell C, D'lima D. 2010. An in vivo evaluation of bone response to three implant surfaces using a rabbit intramedullary rod model. J Orth Surg Res 5:57-57.

28. Sandan B, Olerud C, Larsson S. 2001. Hydroxyapatite coating enhances fixation of loaded pediclescrews: a mechanical in vivo study in sheep. Eur Spine J 10:334-339. 
29. Anil S, Anand P, Alghamdi H, Jansen J. 2011. Dental Implant Surface Enhancement and Osseointegration. Implant Dentistry - a rapidly evolving practice, Rijeka, InTech 86-90.

30. Stangl R, Pries A, Loos B, Mãfaller M, Erben RG. 2004. Influence of pores created by laser superfinishing on osseointegration of titanium alloy implants. J Biomed Mater Res-A 69:444-453.

31. Prodanov L, Lamers E, Wolke J, Huiberts R, Jansen JA, Walboomers XF. 2014. In vivo comparison between laser-treated and grit blasted/acid etched titanium. Clin Oral Implants Res 25(2):234-9.

32. Branemark R, Palmquist A, Lindberg F, Emanuelsson L, Engqvist HK, Thomsen, P. 2010. Biomechanical, histological, and ultrastructural analyses of laser micro- and nanostructured titanium alloy implants: a study in rabbit. J Biomed Mater Res-A 92:1476-1486.

33. Aparicio C, Padras A, Gil F-J. 2011. In vivo evaluation of micro-rough and bioactive titanium dental implants using histometry and pull-out tests. J Mech Behav Biomed Mater 4:1672-1682.

34. Guo Z, Zhou L, Rong M, Zhu A, Geng H. 2010. Bone response to a pure titanium implant surface modified by laser etching and microarc oxidation. Internat J Oral \& Maxill Impl 25:130-136. 
35. Gotfredsen K, Wennerberg A, Johansson C, Skovgaard LT, Harting-Hansen E. 1995. Anchorage of TiO2-blasted, HA-coated, and machined implants: an experimental study with rabbits. J Biomed Mater Res 29:1223-1231.

36. Wennerberg A, Albrektsson T, Andersson B, Krol JJ. 1995. A histomorphometric and removal torque study of screw-shaped titanium implants with three different surface topographies. Clin Oral Impl Res 6:24-30.

37. Faeda R, Tavares H, Sartori R, Guastaldi A, Marcantonio E. 2009. Evaluation of titanium implants with surface modification by laser beam. Biomechanical study in rabbit tibias. Braz Oral Res 23:137-143.

38. Palmquist A, Johansson A, Suska F, Brãnemark R, Thomsen P. 2013. Acute Inflammatory Response to Laser-Induced Micro- and Nano-Sized Titanium Surface Features. Clin Impl Dent Rel Res 15:96-104.

39. Pebe P, Barbot R, Trinidad J, Pesquera A, Lucente J, Nishimura R, Nasr H. 1997. Countertorque testing and histomorphometric analysis of various implant surfaces in canines: a pilot study. Implant Dent 6:259-265.

40. Wong M, Eulenberger J, Schenk R, Hunziker E. 1995. Effect of surface topology on the osseointegration of implant materials in trabecular bone. J Biomed Mater Res 29:1567-1575.

41. Davies JE. 1998. Mechanisms of endosseous integration. Int J Prosthod 11:391-401. 
42. Sinjari B, Guarnieri S, Diomede F, Merciaro I, Mariggio MA, Caputi S, Trubiani O. 2012. Influence of titanium laser surface geometry on proliferation and on morphological features of human mandibular primary osteoblasts. J Biol Reg Homeo Agents 26:505-513.

43. Cook SD, Thomas KA, Kay JF, Jarcho M. 1998. Hydroxyapatite-coated titanium for orthopaedic implant applications. Clin Orth Relat Res 232:225-43.

44. Muller M, Henning FF, Holthorn T, Stangl R. 2006. Bone-implant interface shear modulus and ultimate stress in a transcortical rabbit model of open-pore Ti6Al4V implants. J Biomech 39(11):2123-32.

\section{Figure Legends}

Figure 1.

A photograph showing the positioning of the four implantation sites along the diaphysis of the tibia.

\section{Figure 2.}

Photomicrographs taken using scanning electron microscopy showing [A] the laser textured surface, $[\mathrm{B}]$ an untreated Ti6Al4V surface, $[\mathrm{C}]$ a plasma sprayed hydroxyapatite coated surface and [D] the grit blasted surface.

\section{Figure 3.}

A box-plot illustrating bone apposition rates ( $\mu \mathrm{m} /$ day) between in each of the experimental groups investigated.

\section{Figure 4.}


A box-plot showing bone-implant contact in each of the implant treatment groups.

\section{Figure 5.}

An electronmicrograph showing bone growth to a MF pin [A] and LT pin surface [B].

\section{Figure 6.}

A box-plot of ultimate interfacial shear strength in each of the experimental groups.

\section{Figure 7.}

A photograph showing HA coating delamination following mechanical testing.

\section{Figures 8a and b.}

Scatterplots showing the relationship between mean BIC and Interfacial Shear Strength.

\section{Figure 9.}

Cross-sectional micrographs of samples along the implant interface, (a) HA, (b) LT, (c) GB and (d) MF. Blue arrow indicates new bone growth, yellow arrow shows fibrous tissue in contact with the implant interface. 